\title{
Impactos potenciais das mudanças propostas no Código Florestal Brasileiro sobre as borboletas
}

\author{
André Victor Lucci Freitas ${ }^{1,2}$ \\ ${ }^{1}$ Departamento de Biologia Animal e Museu de Zoologia, Instituto de Biologia, \\ Universidade Estadual de Campinas - UNICAMP, CP 6109, CEP 13083-970, Campinas, SP, Brasil \\ ${ }^{2}$ Autor para correspondência: André Victor Lucci Freitas, e-mail: baku@unicamp.br
}

FREITAS, A.V.L. Potential impacts of the proposed Brazilian Forest Act on native butterflies. Biota Neotrop. 10(4): http://www.biotaneotropica.org.br/v10n4/en/abstract?article+bn00810042010.

\begin{abstract}
This paper discusses how the proposed changes in the Brazilian Forest Act could affect native butterflies. As showed, the main impacts include the reduction in the riparian forests (with consequent loss of connectivity) and the major changes that could take place in the large areas of montane forest. Moreover, changes in the regional landscape, with the reduction of permanently protected areas (Brazilian APPs) and the destruction of hilltop habitats could markedly affect extant butterfly assemblages, which have been severely modified in the past 500 years of deforestation and degradation in all Brazilian habitats.
\end{abstract}

Keywords: conservation, connectivity, Lepidoptera, endangered species.

FREITAS, A.V.L. Impactos potenciais das mudanças propostas no Código Florestal Brasileiro sobre as borboletas. Biota Neotrop. 10(4): http://www.biotaneotropica.org.br/v10n4/pt/abstract?article+bn00810042010.

Resumo: Este trabalho discute como as mudanças propostas no Código Florestal Brasileiro podem afetar as borboletas do Brasil. Conforme exposto, os impactos principais estão na redução das matas ciliares (com consequente perda de conectividade) e nas alterações maiores nas amplas áreas de florestas de altitude. Adicionalmente, alterações na paisagem regional, com redução das áreas de proteção permanentes (APPS) e conversão de topos de morros, por exemplo, podem refletir em profundas mudanças nas assembleias de borboletas já severamente modificadas por quase 500 anos de desmatamento e degradação dos habitats do Brasil.

Palavras-chave: conservação, conectividade, Lepidoptera, espécies ameaçadas.

\section{Introdução}

Por borboletas chamamos as espécies de seis famílias da ordem Lepidoptera, conhecidas por suas asas coloridas e seus hábitos diurnos. Apesar de não proverem serviços ecossistêmicos evidentes, trabalhos recentes mostram que assembleias de borboletas tropicais mudam sua estrutura e composição como resposta a fragmentação, perda de habitat, efeito de borda entre diversos outros tipos de perturbação (DeVries et al. 1997, Ramos 2000, Barlow et al. 2007, Uehara-Prado et al. 2007, Uehara-Prado \& Freitas 2009). Além disso, borboletas são insetos carismáticos e podem ser utilizadas como espécies bandeira e espécies guarda-chuva para conservação (New 1997a, b).

Borboletas apresentam ciclos complexos (lagartas e adultos sofrem pressões distintas) e relativamente curtos (se desenvolvem em poucas semanas), são facilmente observadas e amostradas, podendo ser estudadas com facilidade por métodos padronizados (Freitas et al. 2003, Uehara-Prado et al. 2007). Com o avanço das pesquisas em Biologia da Conservação, nos últimos anos as borboletas conquistaram um lugar de destaque como indicadores biológicos, dada sua sensibilidade comprovada às mudanças ambientais mesmo que muito sutis. Por este motivo, borboletas têm sido usadas cada vez mais em diagnósticos rápidos, estudos comparativos, relatórios de impacto ambiental e monitoramento (Brown 1991, Freitas et al. 2006).

Na maior parte do Brasil e especialmente no Estado de São Paulo, os habitats originais foram drasticamente reduzidos e modificados desde a chegada dos europeus no século XVI. Como resultado, apenas uma pequena fração da Mata Atlântica, e uma menor ainda dos cerrados ainda persistem, geralmente na forma de pequenos fragmentos, muitas vezes bastante perturbados e descaracterizados. Sendo assim, podemos concluir que as comunidades de borboletas que persistem nestes remanescentes são resultantes de um novo equilíbrio alcançado após a conversão destes habitats. Mais ainda, em muitos casos, as populações persistentes devem estar em um limiar de sua manutenção, e alterações mínimas nos habitats remanescentes podem levar muitas destas espécies à extinção local (o que no caso das espécies ameaçadas pode ser bastante crítico).

Com base nas evidências acima, é bastante razoável supor que muitas perdas deverão ocorrer com a supressão de quaisquer porções adicionais de habitat no país. Isso se torna especialmente crítico em biomas já muito reduzidos e alterados, como as matas semidecíduas do interior, a mata atlântica do nordeste, as restingas costeiras, o pampa, e no cada vez mais ameaçado cerrado.

Neste trabalho, pretende-se realizar uma previsão bastante conservadora dos possíveis efeitos das alterações propostas pelo novo código florestal levando-se em conta os dados disponíveis principalmente para borboletas do Brasil. De modo geral, os impactos maiores devem vir das mudanças nas definições das Áreas de Preservação Permanente/APPs, especialmente no que diz respeito às matas ciliares, topos de morros e áreas acima de $1800 \mathrm{~m}$ de altitude, e na redefinição do cômputo da Reserva Legal (RL). 


\section{As Matas Ciliares e Conectividade da Paisagem}

Não há dúvidas de que matas ciliares são um componente importante da paisagem regional no que diz respeito à conservação. É amplamente sabido que as matas ciliares possuem importância não apenas por sua área efetiva, mas também funcionam como corredores importantes de fauna, ligando diferentes fragmentos de habitat e aumentando de forma efetiva a percolação e a conectividade da paisagem (Metzger 2010). Alterações nas matas ciliares ao longo dos rios podem interferir fortemente em assembleias de borboletas neotropicais (Brown 2000), tanto como resultado da perda de habitat quanto pela diminuição da conectividade da paisagem (Brown \& Freitas 2003).

Durante os inventários do projeto "Lepidoptera do Estado de São Paulo" do Programa Biota Fapesp, muitas populações remanescentes de espécies previamente não registradas para o estado foram descobertas em matas ciliares muitas vezes estreitas e muito alteradas. Como exemplo, podemos citar Amphidecta reynoldsi (Nymphalidae) (Figura 1), com uma das duas populações conhecidas para o Estado de São Paulo registrada nas matas ciliares já muito degradadas do Rio Capivari, em Monte Mor. Outro exemplo mais emblemático é o de Parides panthonus castilhoi (Papilionidae) (Brown \& Freitas 2008). Esta borboleta criticamente ameaçada é conhecida apenas de uma área de várzea do Rio Grande próximo à divisa de São Paulo com o Mato Grosso do Sul (esta última de fato registrada pelo Dr. Keith S. Brown nos anos 90, ver Tyler et al. 1994). Em Minas Gerais, Parides burchellanus (Papilionidae) (Figura 1), uma espécie ameaçada, tem poucas populações conhecidas em algumas estreitas matas ciliares nos arredores de Belo Horizonte (Mielke et al. 2004, Mielke \& Casagrande 2008b, AVLF obs. pess.). Ainda não existem estratégias definidas para a conservação de $P$. burchellanus, sendo que as populações de Brumadinho, por exemplo, persistem em matas ciliares muito estreitas que estão ladeadas por pastos e plantações, estando assim muito propensas a desaparecer ante a menor perturbação. Mesmo se a porção remanescente ainda possa parecer adequada, o efeito de borda subsequente pode se encarregar de modificar os habitats remanescentes, levando à extinção completa de algumas populações.

Além de casos específicos, como os dois citados acima, a simples quebra da conectividade pode ser um fator crucial no isolamento e consequente extinção de populações de diversas espécies relativamente comuns de borboletas, especialmente os representantes das tribos Heliconiini (Nymphalide: Heliconiinae) e Ithomiini (Nymphalidae: Danainae) (Brown 2000). Estes dois grupos são compostos por muitas espécies ligadas a florestas (Brown 1979), e suas populações já muito reduzidas pela fragmentação podem estar no limiar da extinção em grande parte de suas distribuições (exceto talvez nas amplas áreas da Amazônia e no corredor da Serra do Mar no Sudeste do Brasil).

Nos casos citados acima e em muitos outros, a redução das matas ciliares pode levar à extinção de populações de diversas espécies localmente. De modo geral, uma das consequências do código ambiental pode ser o início de uma onda de extinções em nosso país, desta vez afetando muitos insetos, e não apenas aves e mamíferos.

\section{As Florestas de Altitude do Sul e Sudeste do Brasil}

Dentre os maiores remanescentes de Mata Atlântica no Sudeste e Sul do Brasil estão as florestas ao longo das serras costeiras (Serra do Mar e Serra Geral) e na Mantiqueira (Tabarelli et al. 2005). Inicialmente pouco exploradas devido à relativa inacessibilidade $\mathrm{e}$ baixo talento para agricultura, áreas extensas de floresta de altitude foram perdidas recentemente devido ao avanço das áreas de ocupação irregular, e nas regiões mais altas pelo avanço da ocupação pelo setor de turismo e hotelaria (especialmente na Serra gaúcha e na Mantiqueira). Mesmo assim, áreas extensas ainda estão relativamente bem preservadas e conectadas nas maiores altitudes das Serras do Mar e da Mantiqueira, abrigando 20 das 55 espécies de borboletas ameaçadas do país. Dentre estas áreas, destacam-se toda a região de Campos do Jordão, o Parque Nacional do Itatiaia, e o Parque Nacional da Serra da Bocaina. Outras áreas ainda pouco conhecidas com extensas áreas de grande altitude (acima de $1400 \mathrm{~m}$ ) incluem o Parque Nacional da Serra do Caparaó na divisa de Minas Gerais com o Espírito Santo e o Parque Nacional do Pico da Neblina na divisa do Amazonas com a Venezuela.

Adicionalmente, estas regiões de grande altitude ainda abrigam um alto número de espécies de borboletas desconhecidas pela ciência. Como exemplo, só na família Nymphalidae pode se citar Eutresis hyperea imeriensis, descrita para o Pico da Neblina (Brown 1977), Yphthimoides cipoensis (Nymphalidae: Satyrinae), possivelmente endêmica da Serra do Cipó (Freitas 2004), Moneuptychia itapeva (Figura 1), descrita de áreas entre 1400 a 2000 m nas Serras da Mantiqueira e do Espinhaço (Freitas 2007), e um novo gênero e espécie, dos Campos de Cima, nas serras altas do Paraná, Santa Catarina e Rio Grande do Sul (Freitas et al. no prelo). Diversas espécies novas já foram coletadas nestes ambientes e aguardam descrição, incluindo mais oito espécies da subfamília Satyrinae, duas espécies de Actinote (Nymphalidae), e certamente inúmeras outras, especialmente nas famílias Hesperiidae, Lycaenidae e Riodinidae ainda aguardam ser descobertas e posteriormente descritas. Muitas destas espécies novas estão sendo descobertas fora de UCs, e algumas delas de fato já podem estar sofrendo efeito da perda de habitat, se encaixando em diversas categorias de ameaça. Como exemplo pode se citar o Actinote eberti (Nymphalidae), em cuja descrição já existe sua proposição como espécie ameaçada (Francini et al. 2004).

Vale a pena ressaltar que, apesar das UCs realmente servirem para proteção efetiva de diversas espécies ameaçadas (só Parque Nacional de Itatiaia abriga 10 espécies de borboletas da fauna ameaçada, dados compilados de Machado et al. 2008), a maioria das espécies da lista das ameaçadas que ocorrem em grandes altitudes possuem populações importantes fora de UCs, coincidindo com as extensas áreas protegidas como Áreas de Preservação Permanente/ APPs. Para exemplificar a importância dessas áreas, na região de Campos de Jordão, os inventários dos últimos 10 anos registraram populações de diversas espécies ameaçadas fora das Unidades de Conservação (UCs). Dentre estas podem se destacar os ninfalídeos Actinote quadra (Figura 1) e Polygrapha suprema, e o riodinídeo Voltinia sanarita (Figura 1) (Freitas \& Brown 2008a, d, Mielke \& Casagrande 2008c, Freitas et al. 2009, AVLF dados não publicados). Mesmo em altitudes menores na Serra do Mar, muitas espécies estão seriamente ameaçadas, como por exemplo Actinote zikani (Figura 1), conhecida hoje de uma única localidade próximo à cidade de São Paulo (Francini et al. 2005, Freitas \& Brown 2008b).

A mudança na legislação em relação às APPs pode levar em médio prazo a uma ocupação desmedida de amplas áreas de florestas de altitude, levando à extinção populações de muitas espécies de borboletas ameaçadas. Em longo prazo pode-se prever que inúmeras espécies hoje não ameaçadas devem entrar na lista, aumentando de forma substancial o número de espécies ameaçadas no Brasil.

\section{Topos de Morros e Biodiversidade Local}

Topos de morros são ambientes muito particulares, e do ponto de vista biológico podem ser considerados consideravelmente diferentes dos habitats vizinhos, mesmo que contíguos, e sua manutenção como APPs é justificada por razões diversas, incluindo proteção de encostas, proteção de cabeceiras de rios e manutenção da diversidade biológica 


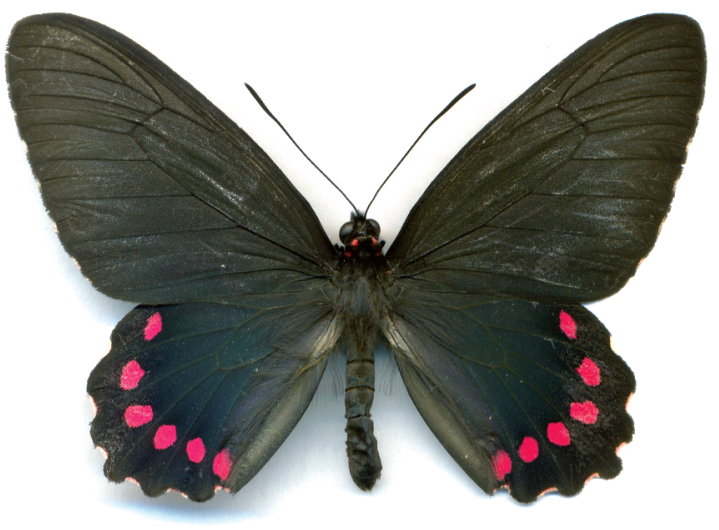

Parides burchellanus

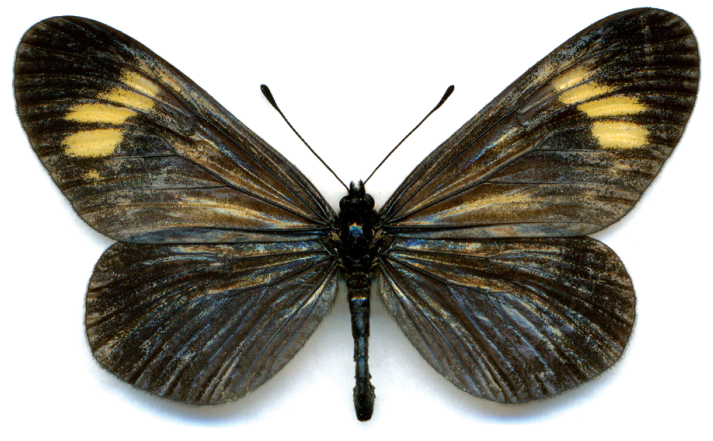

Actinote zikani

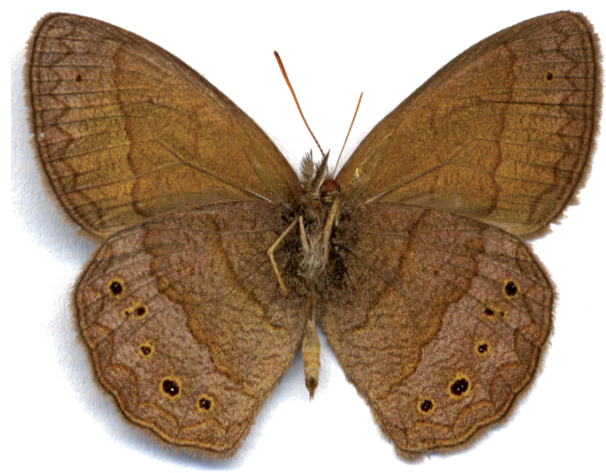

Moneuptychia itapeva

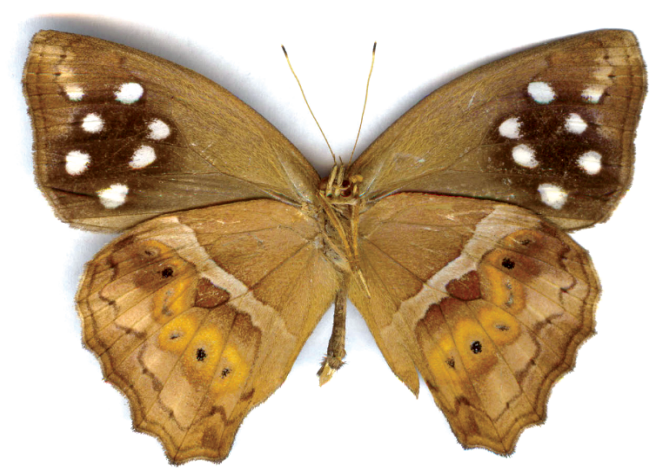

Amphidecta reynoldsi

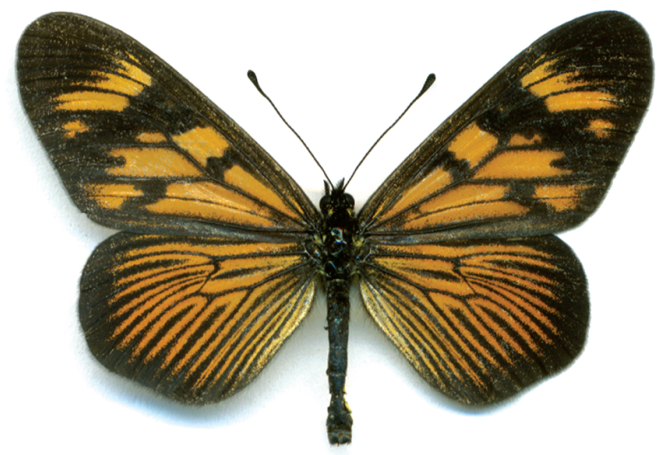

Actinote quadra

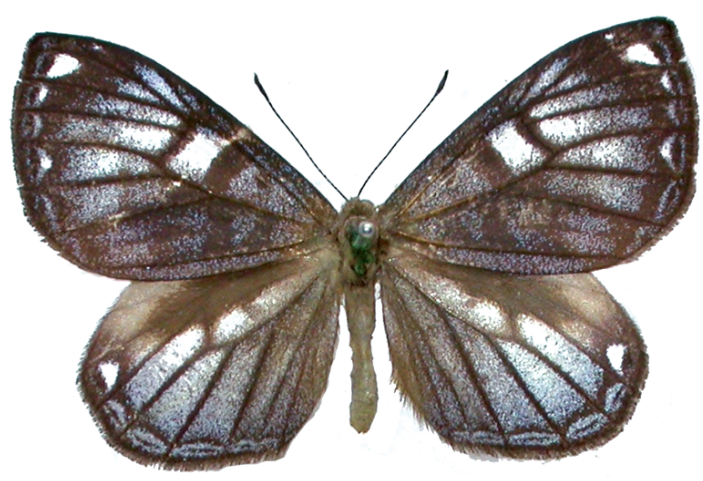

Voltinia sanarita

Figura 1. Seis espécies de borboletas que podem ser afetadas diretamente pelas mudanças propostas no Código Florestal (para maiores detalhes, veja o texto). Figure 1. Six butterfly species that can be directly affected by the proposed changes in the Brazilian Forest Act (for further details see text).

(Metzger 2010). Para borboletas em especial, topos de morros são pontos de encontro para acasalamento para muitas espécies (Baker 1983), sendo ambientes importantes para a manutenção de processos diversos ligados ao reconhecimento intraespecífico, corte e acasalamento.

Diversas espécies de borboletas tem sido registradas apenas em topos de morro, e um exemplo interessante é o hesperídeo ameaçado Zonia zonia diabo, conhecido de poucas localidades no Brasil, a mais consistente sendo o topo do Morro do Diabo, no Parque Estadual do Morro do Diabo, em São Paulo (Mielke \& Casagrande 2008a). Outro caso é a também ameaçada Caenoptychia boulleti (Nymphalidae), cuja maioria dos registros recentes foi feita em topos de morros (Freitas \& Brown 2008c). Entretanto, em parte dos casos é possível que os topos de morro sejam apenas locais de fácil visualização de uma determinada espécie, e não áreas onde as populações são mantidas. 
Ainda assim, existem evidências de que algumas espécies raras mantêm suas populações principalmente em topos de morros. Este parece ser o caso de Agrias claudina (Nymphalidae), com populações perenes e estáveis ocupando alguns topos de morro na região de Joinville, em Santa Catarina. Informações confiáveis do criador de borboletas Herbet Miers, de Joinville, indicam que algumas dessas populações já persistiam a mais de 50 anos (H. Miers 1994 com. pess.), e que as fêmeas colocam seus ovos preferencialmente em plantas presentes próximas ao topo.

Em alguns casos, já foram registradas extinções locais de algumas espécies como resultado de desmatamento e ocupação dessas áreas de topos de morro. No Estado do Rio de Janeiro, a única população conhecida de Heliconius melpomene nanna (Nymphalidae), no limite sul de sua distribuição geográfica (Brown 1979) foi presumivelmente extinta com o avanço da urbanização no Morro da Rocinha (K. S. Brown, com. pess.). Apesar desta borboleta não estar ameaçada, o exemplo ilustra bem que extinções locais podem ocorrer facilmente nos limites de distribuição de uma espécie, onde estas estão possivelmente se mantendo longe das condições ótimas para a espécie.

Um segundo caso bem documentado é da extinção local da ameaçada Caenoptychia boulleti (Nymphalidae) em Petrópolis, no Estado do Rio de Janeiro. Uma das poucas populações conhecidas desta espécie ocorria no topo do Morro Independência, tendo desaparecido com a ocupação do morro por habitações no final dos anos 80 do século passado.

Estes são apenas dois casos de extinção documentada em meio a centenas de outros que devem ter ocorrido nas últimas décadas longe dos olhos dos cientistas. Com a mudança proposta na legislação, é bem provável que muitos outros casos se juntarão aos já verificados, e diversas espécies serão afetadas em maior ou menor grau.

\section{As Assembleias de Borboletas como Reflexo da Paisagem Regional}

Nesta seção, discute-se o impacto específico de duas mudanças relativas à definição da área de Reserva Legal: a inclusão das APPs no cômputo das RL e a possibilidade de compensação em áreas distantes desde que dentro de um mesmo bioma.

Cada unidade da paisagem é única e importante para a manutenção da diversidade de uma região. Por exemplo, os habitats nos topos dos morros não são apenas diferentes topograficamente (por estarem sobre os morros), mas suficientemente distintos florística e ecologicamente de outras unidades da paisagem para serem considerados importantes para a manutenção da biodiversidade local (Metzger 2010). O mesmo ocorre com as matas ciliares, matas de encostas, restingas, veredas e outros ambientes. Sendo que heterogeneidade é um dos fatores comprovadamente importantes para manutenção de diversidade local de borboletas (Brown \& Freitas 2000, 2003), fica claro que a manutenção da diversidade de habitats deve ser promovida pela legislação.

Além do problema citado acima, essa mudança aparentemente simples pode se converter em um sério risco em áreas já extremamente desmatadas, como alguns estados do Sudeste do Brasil. Em casos mais extremos, alguns estados podem ser até considerados como tendo um excesso de áreas protegidas. O resultado nestes casos é que apenas a vegetação das UCs e APPs seriam protegidas por lei, e num cenário pessimista, mas não impossível, toda vegetação remanescente seria suprimida. Neste caso, e levando-se em conta os exemplos já citados neste trabalho, a perda de espécies em algumas regiões do país poderia chegar a níveis muito altos, e não previsíveis em sua totalidade, embora incluindo o aumento da vulnerabilidade de muitas espécies ameaçadas.
Por fim, a sugestão de que a compensação ambiental possa ser feita dentro de um mesmo bioma, o que pode significar potencialmente mais de $3000 \mathrm{~km}$ entre a área desmatada e a compensação, pode implicar em perdas irreparáveis de espécies em algumas áreas já criticamente devastadas e ameaçadas.

Em última instância, devido ao grande número de trabalhos focando no potencial de borboletas como bioindicadores, está bem demonstrado que as respostas exibidas por borboletas geralmente são acompanhadas por mudanças equivalentes em muitos outros grupos de organismos (Brown 1991, Freitas et al. 2006, Bonebrack et al. 2010). É bastante claro que uma alta diversidade biológica caracteriza um ambiente saudável, com consequências diretas para a qualidade de vida do ser humano.

Sendo assim, é mais do que razoável se exigir uma legislação que promova a manutenção da diversidade biológica em todos os níveis (local, regional e nacional), através da manutenção dos habitats remanescentes, e com espaço garantido para pressionar para a recuperação quando assim for previsto. Neste sentido, as mudanças propostas no código florestal são um retrocesso no sentido que abrem espaço para diminuição generalizada das florestas, cerrados e outros habitats, com consequências desastrosas não apenas para a biota local (como as borboletas aqui tratadas), mas para o bem estar de toda a sociedade.

\section{Agradecimentos}

Ao Carlos Joly pelo convite para escrever esse artigo e pelas sugestões desde o princípio. Aos colegas Cristiano Agra Iserhard, Thomas M. Lewinsohn e Paulo S. Oliveira pelas críticas e sugestões na versão final do trabalho. Ao CNPq (300282/2008-7), e à FAPESP (auxílios 00/01484-1 e 04/05269-9, e 98/05101-8 do Biota Fapesp) pelo auxílio financeiro.

\section{Referências Bibliográficas}

BAKER, R.R. 1983. Insect Territoriality. Annu. Rev. Entomol. 28:65-89.

BARLOW, J., GARDNER, T.A., ARAUJO, I.S., AVILA-PIRES, T.C., BONALDO, A.B., COSTA, J.E., ESPOSITO, M.C., FERREIRA, L.V., HAWES, J., HERNANDEZ, M.M., HOOGMOED, M.S., LEITE, R.N., LO-MAN-HUNG, N.F., MALCOLM, J.R., MARTINS, M.B., MESTRE, L.A.M., MIRANDA-SANTOS, R., NUNES-GUTJAHR, A.L., OVERAL, W.L., PARRY, L., PETERS, S.L., RIBEIRO-JUNIOR, M.A., DA SILVA, M.N.F., MOTTA, C.S. \& PERES, C.A. 2007. Quantifying the biodiversity value of tropical primary, secondary, and plantation forests. PNAS 104:18555-18560.

BONEBRACK, T.C., PONISIO, L.C., BOGGS, C.L. \& EHRLICH, P.R. 2010. More than just indicators: a review of tropical butterfly ecology and conservation. Biol. Conserv. 143:1831-1841.

BROWN Jr., K.S. \& FREITAS, A.V.L. 2008. Parides panthonus castilhoi D'Almeida, 1967. In Livro vermelho da fauna brasileira ameaçada de extinção(A.B.M. Machado, G. M.M. Drummond \& A.P. Paglia, ed. MMA, Brasília, DF, Fundação Biodiversitas, Belo Horizonte, MG, p.437-438.

BROWN Jr., K.S. \& FREITAS, A.V.L. 2000. Atlantic Forest butterflies: indicators for landscape conservation. Biotropica 32:934-956.

BROWN Jr., K.S. \& FREITAS, A.V.L. 2003. Butterfly communities of urban forest fragments in Campinas, São Paulo, Brazil: structure, instability, environmental correlates, and conservation. J. Insect. Conserv. 6:217231.

BROWN Jr., K.S. 1977. Centros de evolução, refúgios quaternários e conservação de patrimônios genéticos na região neotropical: padrões de diferenciação em Ithomiinae (Lepidoptera: Nymphalidae). Acta Amaz. 7:75-137.

BROWN Jr., K.S. 1979. Ecologia geográfica e evolução nas florestas neotropicais. UNICAMP, Campinas, 120p. 
BROWN Jr., K.S. 1991. Conservation of neotropical environments: insects as indicators. In The conservation of insects and their habitats: $15^{\text {th }}$ Symposium of the Royal Entomological Society of London, 14-15 September 1989 at the Department of Physics Lecture Theatre, Imperial College, London. (N.M. Collins \& J.A. Thomas, ed.). Academic Press, London, p.349-404.

BROWN Jr., K.S. 2000. Insetos indicadores da história, composição, diversidade e integridade de matas ciliares. In: Matas Ciliares: conservação e recuperação. 2. ed. (R.R. Rodrigues \& H.F. Leitão-Filho, ed.). EDUSP, São Paulo, p.223-232.

DEVRIES, P.J., MURRAY, D. \& LANDE, R. 1997. Species diversity in vertical, horizontal, and temporal dimensions of a fruit-feeding butterfly community in an Ecuadorian rainforest. Biol. J. Linn. Soc. 62:343-364.

FRANCINI, R.B., FREITAS, A.V.L. \& BROWN Jr., K.S. 2005. Rediscovery of Actinote zikani (D'Almeida) (Nymphalidae, Heliconiinae, Acraeini): Natural history, population biology and conservation of an endangered butterfly in SE Brazil. J. Lepid. Soc. 59(3):134-142.

FRANCINI, R.B., FREITAS, A.V.L. \& PENZ, C.M. 2004. Two new species of Actinote (Lepidoptera, Nymphalidae) from Southeastern Brazil. Zootaxa 719:1-10.

FREITAS, A. V. L. 2004. A new species of Yphthimoides (Nymphalidae, Satyrinae) from southeastern Brazil. J. Lepid. Soc. 58:7-12.

FREITAS, A.V.L. \& BROWN Jr., K.S. 2008a. Actinote quadra (Schaus, 1902). In Livro vermelho da fauna brasileira ameaçada de extinção (A.B.M. Machado, G.M.M. Drummond \& A.P. Paglia, ed.). MMA, Brasília, DF, Fundação Biodiversitas, Belo Horizonte, MG, p.402-403.

FREITAS, A.V.L. \& BROWN Jr., K.S. 2008b. Actinote zikani D'Almeida, 1951. In: Livro vermelho da fauna brasileira ameaçada de extinção. (A.B.M. Machado, G.M.M. Drummond \& A.P. Paglia, ed.). MMA, Brasília, DF, Fundação Biodiversitas, Belo Horizonte, MG, p.403-404.

FREITAS, A.V.L. \& BROWN Jr., K.S. 2008c. Caenoptychia boulletti Le Cerf, 1919. In Livro vermelho da fauna brasileira ameaçada de extinção. (A.B.M. Machado, G.M.M. Drummond \& A.P. Paglia, ed.). MMA, Brasília, DF, Fundação Biodiversitas, Belo Horizonte, MG, p.404-405.

FREITAS, A.V.L. \& BROWN Jr., K.S. 2008d. Polygrapha suprema (Schaus, 1920). In: Livro vermelho da fauna brasileira ameaçada de extinção. (A.B.M. Machado, G.M.M. Drummond \& A.P. Paglia, ed.). MMA, Brasília, DF, Fundação Biodiversitas, Belo Horizonte, MG, p.425.

FREITAS, A.V.L. 2007. A new species of Moneuptychia Forster (Lepidoptera: Satyrinae: Euptychiina) from the highlands of Southeastern Brazil. Neotrop. Entomol. 36:919-925.

FREITAS, A.V.L., FRANCINI, R.B. \& BROWN Jr., K.S. 2003. Insetos como indicadores ambientais. In Métodos de estudos em biologia da conservação e manejo da vida silvestre. (L. Cullen Jr., R. Rudran \& C. Valladares-Pádua, org.). Editora da UFPR, Fundação O Boticário de Proteção à Natureza, cap.5, p.125-151.

FREITAS, A.V.L., FRANCINI, R.B. \& SOUZA, T.S. 2009. Immature stages and natural history of the threatened butterfly Actinote quadra (Nymphalidae: Heliconiinae: Acraeini). Trop. Lepid. Res. 19(2):82-88.

FREITAS, A.V.L., LEAL, I.R., UEHARA-PRADO, M. \& IANNUZZI, L. 2006. Insetos como indicadores de conservação da paisagem. In: Biologia da conservação: essências. (C.F.D. Rocha, H.G. Bergallo, M. Van Sluys \&. M.A.S. Alves, ed.). RiMa Editora, São Carlos, cap.15, p.357-384.
FREITAS, A.V.L., MIELKE, O.H.H., MOSER, A., SILVA-BRANDÃO, K.L. \& ISERHARD, C.A. no prelo. A new genus and species of Euptychiina (Lepidoptera: Nymphalidae, Satyrinae) from south Brazil. Neotrop. Entomol.

MACHADO, A.B., DRUMMOND, G.M. \& PAGLIA, A.P. (Org.). 2008. Livro vermelho da fauna brasileira ameaçada de extinção. Ministério do Meio Ambiente, Brasília, DF, v.2., 1420p.

METZGER, J.P. 2010. O Código Florestal tem base científica? Nat. \& Conserv. 8:1-5.

MIELKE, O.H.H. \& CASAGRANDE, M.M. 2008a. Zonia zonia diabo Mielke \& Casagrande 1998. In Livro vermelho da fauna brasileira ameaçada de extinção. (A.B.M. Machado, G.M.M. Drummond \& A.P. Paglia, ed.) MMA, Brasília, DF, Fundação Biodiversitas, Belo Horizonte, MG, p.399-400.

MIELKE, O.H.H. \& CASAGRANDE, M.M. 2008b. Parides burchellanus (Westwood, 1872). In Livro vermelho da fauna brasileira ameaçada de extinção. (A.B.M. Machado, G.M.M. Drummond \& A.P. Paglia, ed.). MMA, Brasília, DF, Belo Horizonte, MG, Fundação Biodiversitas, p.435-436.

MIELKE, O.H.H. \& CASAGRANDE, M.M. 2008c. Eucorna sanarita (Schaus, 1902). In Livro vermelho da fauna brasileira ameaçada de extinção. (A.B.M. Machado, G.M.M. Drummond \& A.P. Paglia, ed.). MMA, Brasília, DF, Belo Horizonte, MG, Fundação Biodiversitas, p. 443-444.

MIELKE, O.H.H., MIELKE, C.G. \& CASAGRANDE, M.M. 2004. Parides panthonus jaguarae (Foetterle) redescoberto em Minas Gerais: sua identidade (Lepidoptera: Papilionidae). Rev. Bras. Zool. 21(1):9-12.

NEW, T.R. 1997a. Butterfly conservation. $2^{\text {nd }}$ ed. Oxford University Press, Oxford.

NEW, T.R. 1997b. Are Lepidoptera an effective 'umbrella group'for biodiversity conservation? J. Insect Conserv. 1:5-12.

RAMOS, F.A. 2000. Nymphalid butterfly communities in an amazonian forest fragment. J. Res. Lepid. 35:29-41.

TABARELLI, M., PINTO, L.P., SILVA, J.M.C. \& HIROTA, M. 2005. Challenges and opportunities for biodiversity conservation in the Brazilian Atlantic Forest. Conserv. Biol. 19:695-700.

TYLER, H., BROWN Jr., K.S. \& WILSON, K. 1994. Swallowtail Butterflies of the Americas - a study in biological dynamics, ecological diversity, biosystematics, and conservation. Scientific Publishers, Gainesville, FL.

UEHARA-PRADO, M. \& FREITAS, A.V.L. 2009. The effect of rainforest fragmentation on species diversity and mimicry ring composition of ithomiine butterflies. Insect Conserv. Div. 2:23-28.

UEHARA-PRADO, M., BROWN Jr., K.S. \& FREITAS, A.V.L., 2007. Species richness, composition and abundance of fruit-feeding butterflies in the Brazilian Atlantic Forest: comparison between a fragmented and a continuous landscape. Global Ecol. Biogeogr. 16:43-54.

UEHARA-PRADO, M., FERNANDES, J.O., BELLO, A.M., MACHADO, G., SANTOS, A.J., VAZ-DE-MELLO, F.Z. \& FREITAS, A.V.L. 2009. Selecting terrestrial arthropods as indicators of small-scale disturbance: A first approach in the Brazilian Atlantic Forest. Biol. Conserv. 142:1220-1228. 
